\title{
México, Guatemala y Costa Rica: educación sexual en los países con altas tasas de bullying en Latinoamérica
}

Diogo Cavazotti Aires ${ }^{1}$

\begin{abstract}
Resumen:
El estudio investiga cómo México, Guatemala y Costa Rica trabajan la educación sexual basada en las leyes nacionales vigentes. El objetivo es analizar si estos países siguen las recomendaciones definidas en los convenios internacionales de derechos humanos, que desde la década de 1990 han abordado la importancia de la educación sexual como una forma de combatir diversos problemas sociales, incluido el bullying (también abordado por la literatura como acoso escolar). Las tres naciones investigadas están incluidas en la lista más reciente de los 25 países del mundo con más casos de acoso escolar, realizada por la Organización para la Cooperación y el Desarrollo Económico (OCDE) y por la ONG Internacional Bullying Sin Fronteras. A partir de la Declaración Universal de los Derechos Humanos hasta documentos más recientes, la educación fue tratada como prioridad en la infancia y la adolescencia, con el objetivo de promover el desarrollo de la personalidad integral del ser humano. Esto incluye la atención a la enseñanza de la sexualidad y también para mejorar las condiciones y el acceso a la salud pública, al crecimiento personal, profesional y económico y para la resolución de problemas sociales.
\end{abstract}

\section{Palabras clave:}

Educación sexual. Convenciones internacionales. Bullying. Derechos humanos. América Latina.

\section{México, Guatemala e Costa Rica: educação sexual em países com altas taxas de bullying na América Latina}

\begin{abstract}
Resumo:
O estudo investiga como o México, Guatemala e Costa Rica trabalham a educação sexual com base nas leis nacionais existentes. O objetivo é examinar se esses países seguem as recomendações definidas nas convenções internacionais de direitos humanos, que desde a década de 1990 abordam a importância da educação sexual como forma de combater vários problemas sociais, incluindo o bullying. As três nações investigadas estão incluídas na lista mais recente dos 25 países do mundo com mais casos de bullying, feita pela Organização para a Cooperação e Desenvolvimento Econômico
\end{abstract}

1 Doutorando em Educação pela Universidade do Estado de Santa Catarina. Mestre em Direitos Humanos e Direito Internacional Humanitário pela Universidad Católica de Colombia. Pós-graduando em Educação em Direitos Humanos pela Universidade Federal do ABC. Graduado em Comunicação Social. E-mail: dcavazotti@gmail.com ORCID iD: https://orcid.org/0000-0001-6340-7770 
(OCDE) e pela ONG Internacional Bullying Sem Fronteiras. Da Declaração Universal dos Direitos Humanos até os documentos mais recentes, a educação foi tratada como prioridade em crianças e adolescentes, com o objetivo de promover o desenvolvimento da personalidade integral do ser humano. Isso inclui a atenção ao ensino da sexualidade e também a melhoria das condições e ao acesso à saúde pública, ao crescimento pessoal, profissional e econômico e à resolução de problemas sociais.

Palavras-chave:

Educação sexual. Convenções internacionais. Bullying. Direitos humanos. América Latina.

\section{Mexico, Guatemala and Costa Rica: sex education in countries with high bullying rates in Latin America}

\section{Abstract:}

The study investigates how Mexico, Guatemala and Costa Rica work on sex education based on existing national laws. The objective seeks to analyze whether these countries follow the recommendations defined in international human rights conventions, which since the 1990s have addressed the importance of sex education as a way to battle several problems in society, including bullying (also addressed by literature as bullying). The three investigated nations are included in the most recent list of the 25 countries in the world with the highest cases of bullying, and it was made by the Organization for Economic Co-operation and Development (OECD) and the International NGO Bullying Without Borders. From the Universal Declaration of Human Rights to more recent documents, education was treated as a priority in children and adolescents, with the aim of promoting the development of the integral personality of the human being. This includes attention to the teaching of sexuality and also to improve conditions and access to public health, personal, professional and economic growth and for the resolution of social problems.

\section{Keywords:}

Sex education. International conventions. Bullying. Human rights. Latin America.

\section{Introducción}

Este artículo tiene como objetivo analizar las leyes de educación sexual vigentes en México, Guatemala y Costa Rica de acuerdo con las convenciones internacionales de derechos humanos. El criterio de selección para la elección de estos actores fue la posición de algunos países latinos en el ranking de las 25 naciones con más episodios de bullying en el mundo, realizado por la Organización para la Cooperación y el Desarrollo Económico (OCDE) y por la ONG Internacional Bullying Sin Fronteras. En el estudio, México aparece en la primera posición, Guatemala en noveno y Costa Rica en onceavo (BSF, 2020).

De acuerdo con autores y organismos de derechos humanos acá mencionados, la educación sexual hecha de manera integral y transdisciplinaria logra una serie de beneficios para niños, niñas y adolescentes, incluyendo la disminución de episodios de bullying. Además, desde la década de 1990 la temática de la educación para la sexualidad aparece de manera más efectiva en las convenciones internacionales de derechos humanos, incluso con consejos y métodos de enseñanza.

Este estudio busca identificar si las leyes nacionales de educación sexual en los países mencionados cumplen con los acuerdos internacionales firmados por estas naciones y que abordan la educación sexual para niños, niñas y jóvenes, ya que "los derechos sexuales y reproductivos son parte de los derechos humanos y están protegidos por el derecho internacional" (BEDOYA ABELLA, 2014, p. 96). 
El reconocimiento de la sexualidad como parte fundamental para una educación integral del ciudadano es visto como un gran desafío por diferentes autores, principalmente debido a una resistencia histórica de los Estados. Para Astudillo, la complejidad de abordar este tema radica en el fracaso de los propios Estados para regular la sexualidad humana a través de la educación (ASTUDILLO, 2017).

En el campo educativo el déficit democrático es grande en oportunidades, en cobertura, discriminación de género y en el hecho de negar o guardar información respecto a la sexualidad, razón por la cual en nuestro continente americano la educación sexual sólo por excepción ha sido incluida de manera formal en los programas escolares oficiales. (LONDOÑO, 1996, p. 232).

Por esta dificultad los organismos de derechos humanos pasaron, en las últimas décadas, a prestar más atención al tema en convenciones internacionales, abordando la importancia del asunto en el ámbito educacional, estratégico y de derechos humanos. Cabe aclarar que esta investigación se focaliza en las leyes de educación sexual, y no en leyes o programas específicos sobre el bullying, por comprender, de acuerdo con las fuentes presentadas, que el combate a este problema presente en las escuelas y colegios se realiza de manera horizontal y transdisciplinaria.

México, Guatemala y Costa Rica, que están en posiciones distintas en el ranking, ya reconocen los desafíos que necesitan ser enfrentados para combatir el acoso escolar:

El acoso u hostigamiento escolar, mejor conocido como bullying, es un tema al que se le ha prestado atención recientemente en México con la puesta en marcha de campañas de concientización sobre el fenómeno. Sin embargo, no se han tomado decisiones legislativas claras que permitan el apoyo directo a los afectados y sus familias, dado que, además del bullying por parte de los compañeros de clase, en ocasiones los propios docentes favorecen este tipo de conductas al no estar preparados para enfrentar la situación, lo que deviene en impunidad para el agresor y la consiguiente vejación de la víctima, no sólo en el ámbito escolar, sino también en su propio desarrollo de vida, llegando en ocasiones a causársele incluso la muerte. (PESCI EGUIA, 2015, p. 105).

(...) el acoso afecta aproximadamente al $32 \%$ de los estudiantes del mundo y Guatemala no es la excepción. Según la Encuesta Nacional sobre Violencia y Clima Escolar 2015 - el registro más reciente -, en el país, el 14\% de los estudiantes de primaria y secundaria han sufrido acoso escolar. (MARTÍNEZ; HERRERA, 2020).

El aumento de la violencia y las conductas agresivas tendientes a la destructividad humana ha ido alcanzando niveles insospechados en el presente siglo en el ámbito internacional y Costa Rica no ha sido la excepción. Esta se ha constituido en una de las problemáticas más complicadas de resolver, en la medida que promueve la instauración de una cultura basada en la violencia que incide negativamente en todos los actores involucrados, en tanto la reiterada exposición a la violencia conlleva a la habituación y al riesgo de considerarla normal o aún peor, inevitable. (MORALES-RAMIREZ; VILLALOBOS-CORDERO, 2017, p. 04).

\section{Metodología}

El análisis se llevó a cabo mediante el cruce de datos con base en los documentos de las convenciones internacionales de derechos humanos reconocidas en los países analizados y las leyes vigentes que abordan educación sexual en las mismas naciones. A través de la indagación de fuentes primarias (origen de la información) y secundarias (análisis e interpretaciones), se buscó establecer los cimientos necesarios que colocan la educación sexual en un espacio especial dentro 
del campo de los derechos humanos. Así, de manera cualitativa, el estudio plantea aspectos constitucionales y jurídicos que ponen a estos países bien sea en acuerdo, o en desacuerdo, en relación con las recomendaciones emitidas por organismos internacionales de derechos humanos. La elección de un análisis documental de México, Guatemala y Costa Rica se hizo para establecer cómo estos países trabajan la educación en sexualidad en leyes nacionales. Según los autores analizados, esta es, entre otros, una de las formas de disminuir casos de bullying dentro de la cultura latina. La revisión de la literatura reúne a autores y autoras de diferentes áreas del conocimiento con el fin de mostrar cómo el tema de la educación sexual es transdisciplinario, es decir, tiene diferentes áreas involucradas. De este modo, se buscaron puntos de referencia en los estudios del derecho, la psicología, la educación, la sexualidad, los derechos humanos, y la ciudadanía.

\section{Bullying y sus consecuencias}

El ser humano es agregado por similares, con diferentes estilos y principios. Y a través de estos grupos adquiere conciencia de sí mismo y del otro. El problema ocurre cuando hay falta de respeto por las diferencias, generando diversas formas de agresividad (MEIER; ROLIM, 2013). Dentro de esta realidad, Olweus (1993) presenta características frecuentes en episodios de acoso: acciones constantes, intención de provocar dolor físico o emocional y que el agresor tenga mayor poder en relación con la víctima.

Un estudio de 2010 realizado en 16 países de América Latina, incluso los acá analizados, encontró que los niños acosados en entornos escolares sufren significativamente menor rendimiento de lectura y matemáticas en comparación con los otros (COLOMBIA, 2018), limitando así, a futuro, incluso las oportunidades intelectuales y laborales.

El alto nivel de estrés que enfrenta una víctima de acoso también puede causar otros problemas. Entre las posibles patologías, las más comunes son diarrea, mareos, dolor de cabeza y de estómago, fiebre, dolores musculares, úlceras, gastritis, anorexia, bulimia, obesidad, alergias y problemas respiratorios en general (MEIER; ROLIM, 2013).

En el campo de la psiquiatría, también no son pocas las consecuencias posibles, que incluyen trastornos del estado de ánimo, depresión, alta ansiedad y fobias. Además, existe la incidencia de problemas psicológicos como baja autoestima, inseguridad, introversión exacerbada y pensamientos suicidas. (MEIER; ROLIM, 2013, p. 56-57).

Algunos de estos pensamientos se materializan. Apenas un ejemplo: en 2014, un joven colombiano de 16 años se suicidó después de sufrir perjuicios en la escuela secundaria. Le tomaron una foto junto a su novio y se la mostraron a los maestros. La víctima se lanzó del piso más alto de un centro comercial y generó un intenso debate en todo el país (DURÁN, 2014). El desafío es grande, considerando que uno de cada tres adolescentes sufre acoso escolar en el mundo (UNESCO, 2018b) y que los suicidios, en muchos casos, también se producen en momentos de impulsividad, frente a conflictos, violencia, abusos y sensaciones de aislamiento (OMS, 2019).

Entre tanto, los problemas no se quedan presentes solo en la infancia y adolescencia, reproduciendo el sufrimiento y causando estragos en la salud y en la obtención de oportunidades laborales.

En la edad adulta, las víctimas de acoso escolar pueden tener dificultades para aceptar nuevos desafíos, como promociones o participar en nuevas empresas. (...) En la vida personal, una posible consecuencia es el desarrollo de una introspección exagerada, casi un aislamiento social, que a menudo ocurre inconscientemente (...). (MEIER; ROLIM, 2013, p. 57-58). 


\section{Beneficios del acceso a la educación sexual}

Las Directrices Técnicas Internacionales sobre Educación Sexual de la Organización de las Naciones Unidas para la Educación, la Ciencia y la Cultura (UNESCO) muestran que la educación sexual es un importante elemento para la prevención de las infecciones de transmisión sexual (ITS), la reducción de los episodios de embarazo adolescente, contribuye a retrasar el inicio de la vida sexual, reducir los casos coercitivos de violencia dentro de este tema, incluso el bullying, así como otras vulnerabilidades que enfrentan los niños, niñas y adolescentes. La educación sexual también representa una estrategia hacia los Objetivos de Desarrollo del Milenio (ODM), incluso para lograr la igualdad de género (UNESCO, 2010).

Del mismo modo, los expertos dicen que la educación en igualdad de género es esencial para prevenir la violencia, tanto en niñas, niños, adolescentes, mujeres y contra la comunidad LGBTQI+. "Es imposible alcanzar niveles razonables de violencia sin que, en las escuelas, desde la educación de la primera infancia, haya una preparación" (FRISCHEISEN, 2018), es decir, un enfoque sobre este tema bajo el sesgo de los derechos humanos y la ciencia, antes del acceso a los comportamientos culturales y sociales, para disminuir la intolerancia a la diferencia.

La educación de los jóvenes es la única solución sostenible y verdadera contra la violencia por razones de género. (...) Las escuelas pueden desempeñar un papel esencial para erradicar la violencia por razones de género. (...) las escuelas deben aplicar políticas de tolerancia cero en materia de acoso sexual y de intimidación, fundamentalmente en cuestiones de estigmatización y discriminación basadas en la orientación sexual y la identidad de género. (UNESCO, 2018a).

Para promover esta atención, las Naciones Unidas han elaborado instrucciones sobre cómo abordar la educación sexual. El enfoque debe incluir las cuestiones de diversidad y género como una forma de promover el respeto. Por otra parte, los padres deben ser incluidos para que el proceso de aprendizaje y conocimiento continúe en casa. Además, la educación sexual también puede combinarse con otras disciplinas de manera transversal (ONU, 2018).

El enfoque de educación sexual en derechos humanos problematiza, explicita y trata de deconstruir las representaciones sociales negativas impuestas a los sujetos e identidades excluidos (por clase, raza/etnia, sexo, género, sexualidad, orientación sexual, regionalidad). (ALMEIDA; LUZ, 2014, p. 100).

\section{El derecho humano a la educación sexual}

La educación sexual se hizo más presente en las convenciones internacionales de derechos humanos desde la década de 1990, cuando hubo una mayor atención a la planificación familiar y la salud sexual y reproductiva, con un enfoque en el universo femenino. Al final de la Conferencia Internacional de las Naciones Unidas sobre la Población y el Desarrollo, en 1994, el resultado fue la creación de un plan de compromiso para mejorar la vida de las personas en esta esfera. El documento fue criticado por algunos de los 179 países participantes, pero el tema de la educación sexual fue visto como una de las formas de desarrollar objetivos en las naciones (ONU, 1995). Sawyer (2019, p. 08) afirma que en ese momento "la salud y los derechos reproductivos y sexuales fueron una nueva síntesis entre la planificación familiar y el feminismo, hasta ahora considerados incompatibles".

En 1994, la Convención Interamericana para Prevenir, Sancionar y Erradicar la Violencia contra la Mujer (Convención de Belém do Pará), implanta que los Estados Partes deben adoptar 
programas para promover el derecho a una vida libre de violencia (OEA, 1995). Para eso, también sugiere la educación:

Modificar los patrones socioculturales de conducta de hombres y mujeres, incluyendo el diseño de programas de educación formales y no formales apropiados a todo nivel del proceso educativo, para contrarrestar prejuicios y costumbres y todo otro tipo de prácticas que se basen en la premisa de la inferioridad o superioridad de cualquiera de los géneros o en los papeles estereotipados para el hombre y la mujer que legitiman o exacerban la violencia contra la mujer. (OEA, 1995, p. 3).

Para Spaccarotella (2019), la Convención de Belém do Pará proporcionó una nueva generación de leyes, nacionales e internacionales, además de inspirar el desarrollo de políticas públicas, campañas de servicios dentro de la educación en sexualidad.

$\mathrm{Al}$ año siguiente, durante la Conferencia Internacional sobre la Mujer de Beijing, se propusieron garantías que incluían la educación y la promoción de la salud sexual y reproductiva (ONU, 1996). Una vez más, el tema fue controvertido, especialmente entre los países occidentales donde la religión tiene un carácter de poder político (ALVES, 2001). Sobre todo, la ocasión sirvió como "hito esencial en el proceso de consolidación de los derechos de las mujeres a nivel internacional" (GUARNIERI, 2010, p. 26). Y cuando se trata de los derechos de las mujeres, están sustancialmente incluidos los derechos sexuales de la información y de la educación.

Haciendo un corte histórico y llevando el tema al presente, el informe del Consejo de Derechos Humanos de las Naciones Unidas de 2018 resalta la necesidad de que los países emprendan la igualdad de género y los derechos de las mujeres y las niñas, también en el seno de las familias, al igual que programas de formación de docentes, los cuales aborden la prevención de temas como violencia sexual y de género, "garantizando el acceso universal a una educación sexual integral y con base empírica” (ONU, 2019, p. 157). Además, las estrategias para jóvenes en los Programas de Desarrollo de las Naciones Unidas expresan que "existe una gran necesidad de invertir en educación sexual y reproductiva y servicios de salud especialmente diseñados para los jóvenes” (ONU, 2014, p. 12).

Pero para que hoy las convenciones internacionales de derechos humanos lleguen a debatir esta cuestión, décadas antes se tratara indirectamente. La más conocida, la Declaración Universal de Derechos Humanos de 1948, plantó la educación como objeto de pleno desarrollo de la personalidad humana y que debería favorecer la tolerancia (ONU, 2009).

En el mismo contexto, el Pacto Internacional de Derechos Económicos, Sociales y Culturales aparece en 1966 y explora el tema de la educación donde se abordan temas inherentes a los objetivos alcanzados por medio de la educación sexual:

Los Estados Partes en este Pacto reconocen el derecho de todos a la educación. Coinciden en que la educación debe orientarse al pleno desarrollo de la personalidad humana y al sentido de su dignidad, así como a fortalecer el respeto de los derechos humanos y las libertades fundamentales. (ONU, 1976).

En 1988 surge el Protocolo de San Salvador, adicional a la Convención Americana sobre Derechos Humanos, en materia de derechos económicos, sociales y culturales, para que las naciones colaboren por un hemisferio democrático, de libertad personal y justicia social, basado en el respeto de los derechos humanos. El documento defiende que los países proporcionen educación sobre la prevención y el tratamiento de los problemas de salud. En el Pacto, "la educación también aparece con fuerza como herramienta de difusión y trabajo sobre los objetivos, políticas y programas vinculados con los tratados que persiguen metas concretas" (SCIOSCIOLI, 2014, p. 12). Si no especifica el tema de la educación para la sexualidad, menciona objetivos y metas que se logran a través de este enfoque. 
Dos años después, la Convención sobre los Derechos del Niño, documento de derechos humanos más ratificado en el mundo, aborda los derechos inherentes a la infancia, incluidas las obligaciones comunes a los Estados con respecto a la educación y el desarrollo:

Los Estados Partes adoptarán todas las medidas legislativas, administrativas, sociales y educativas apropiadas para proteger al niño de toda forma de violencia física o mental, delitos o abuso, negligencia o trato irrazonable, malos tratos o explotación, incluido el abuso sexual, mientras el niño esté bajo la custodia de los padres, tutor legal o cualquier otra persona responsable de ello. (UNICEF, 1990).

La importancia práctica del documento es expresada por Bruñol:

Con las leyes de menores, especialmente en América Latina, los niños no fueron suficientemente protegidos de la arbitrariedad privada y quedaron expuestos a diversas formas de abuso público, antes desconocidas, debido a la indiferencia de los órganos del Estado hacia la infancia. Sólo con el proceso iniciado con la Convención (sobre los Derechos del Niño) en el que los intereses de los niños se convierten en genuinos derechos, los niños podrán oponer sus derechos como límite y orientación tanto de la actuación de los padres, como del Estado. (BRUÑOL, 2001, p. 37).

México, Guatemala y Costa Rica adhirieron a las convenciones abordadas en esto estudio.

Actualmente, la Organización de las Naciones Unidas para la Educación, la Ciencia y la Cultura (UNESCO) ha considerado la educación sexual como una forma para que los niños y adolescentes reciban información que permite la toma de decisiones de manera responsable tanto en aspectos de sexualidad como en las relaciones interpersonales:

O dejamos a los niños y niñas encontrar su propio camino en la nebulosa de información parcial, la desinformación y la explotación que podrían encontrar en los medios de comunicación, Internet, grupos de pares o personas inescrupulosas; o aceptamos la tarea de proporcionar una educación en sexualidad clara, informada, sustentada en hechos científicos e inspirada en los valores universales de respeto y derechos humanos. (UNESCO, 2010, p. iii).

Veamos cómo México, Guatemala y Costa Rica trabajan el tema de la educación sexual desde las leyes y planes nacionales vigentes. Con el fin de lograr un espectro más amplio, se presentan, de manera directa y resumida, los extractos donde el tema aparece en los documentos de cada nación.

\section{México}

Ramírez (2004) explica que México fue pionero en América Latina en la enseñanza de la educación sexual. La práctica comenzó en 1974, cuando se introdujeron temas como la reproducción y la pubertad en la educación primaria. En la escuela secundaria, se mencionaron temas como la prevención del embarazo y de infecciones de transmisión sexual. Como resultado, se presenciaron iniciativas negativas, como la quema de libros con contenido educativo del género. Acercando el tema al presente, un estudio de 2017 analizó que en México:

El porcentaje de adolescentes que reportan recibir educación sexual integral varía dependiendo de los temas y nivel escolar. Los temas más frecuentes están relacionados con salud sexual y reproductiva; los menos tratados son derechos y relaciones. Los contenidos de educación sexual se trasmiten mayoritariamente durante la escuela secundaria. (ROJAS et al., 2017, p. 19). 
Un documento oficial que aborda mayoritariamente la enseñanza de la sexualidad en México es de 2013, con actualizaciones recientes, titulado Modelo de Atención Integral Salud Sexual y Reproductiva para Adolescentes, donde existen conceptos, marcos jurídicos, revisión de instrumentos internacionales y un modelo a seguir por instituciones del sistema nacional de salud. El material muestra pautas en cuestiones de salud sexual y reproductiva, pero no un efectivo plan de trabajo (MÉXICO, 2018a).

El texto vigente de la Ley General de Educación habla que temas sobre educación sexual podrán pasar por sugerencias sobre el contenido por parte de la Secretaría de Salud. Del mismo modo, contenido de educación sexual que implica el ejercicio responsable de la sexualidad, la planeación familiar, la maternidad y la paternidad responsable, la prevención de los embarazos adolescentes y de las infecciones de transmisión sexual son temas de responsabilidad del Estado, sus organismos descentralizados y particulares autorizados (MÉXICO, 2019a).

Todavía más reciente, el Programa Sectorial de Educación habla de la necesidad de revisar la educación, entre ellas la sexual, para repercutir la manera como niñas, niños y adolescentes perciben el mundo y se desarrollan (MÉXICO, 2020a).

Otros diversos documentos oficiales de México abordan prácticamente los mismos conceptos de educación sexual, como la Constitución Política de los Estados Unidos Mexicanos, de 1917, pero que en la reforma publicada en 2020 obliga a que los planes y programas de estudio tengan perspectiva de género y la educación en sexualidad (MÉXICO, 2020b). Del mismo modo, la Ley General de los Derechos de Niñas, Niños y Adolescentes, también reformulada, establece que este público tiene derecho de disfrutar los más altos niveles de salud, incluso con orientación sobre la sexualidad (MÉXICO, 2019b). Así como el Plan Nacional de Desarrollo 2019-2024, que visa priorizar la prevención de enfermedades con programas escolares de salud sexual (MÉXICO, 2019c).

Los mismos conceptos están presentes en la Estrategia Nacional de Atención a la Primera Infancia (MÉXICO, 2018b), el Acuerdo n. ${ }^{\circ}$ 592/2011, con articulaciones sobre la educación básica (MÉXICO, 2018c), la Agenda Digital Educativa (MÉXICO, 2019d), la Estrategia Nacional para la Prevención del Embarazo en Adolescentes (MÉXICO, 2018d) y la Ley General de Salud (MÉXICO, 2018e).

Es digno de mención la Estrategia Nacional de Educación Inclusiva, que aborda la necesidad que el sector educativo reciba cursos de formación en diferentes modalidades, incluso la igualdad de género y la educación sexual (MÉXICO, 2018f).

\section{Guatemala}

La Ley de Protección de la Niñez y Adolescencia de Guatemala, diseñado para el desarrollo integral de este público, habla en ejecutar programas de educación sexual para la prevención de enfermedades y para la procreación y la vida en pareja que inculquen la paternidad y maternidad responsables (GUATEMALA, 2018a). En Informe de la Estrategia de Articulación de los Objetivos de Desarrollo Sostenible al Plan y la Política Nacional de Desarrollo K'atun: Nuestra Guatemala al 2032, establece como meta garantizar acceso a la información y a la educación en sexualidad (GUATEMALA, 2018b).

El informe K’atun también elabora políticas de desarrollo y tiene por objetivo contextualizar el país a la agenda internacional. Para eso, propone intensificar acciones para informar, educar y garantizar el acceso a la educación sexual a toda la población para disminuir infecciones de VIH y muertes maternas en poblaciones con elevados niveles de pobreza (GUATEMALA, 2018c). Además, propone una reforma en la enseñanza de este tema con una nueva mirada:

La educación sexual integral debe conducir a tomar decisiones con información veraz, pertinente y oportuna; posibilita el desarrollo de una cultura de responsabilidad, así como el ejercicio de 
derechos al respecto de una vida sexual satisfactoria y sin riesgos, con la suficiente libertad para decidir cuándo y con qué frecuencia procrear o no. (GUATEMALA, 2018c, p. 160).

Hay garantías de educación sexual en el sistema educativo con pertinencia cultural, científica y humanística en la Política Nacional de Promoción y Desarrollo Integral de las Mujeres y Plan de Equidad de Oportunidades 2008-2023. Para eso propone "crear e implementar programas de formación para las y los docentes del sistema educativo nacional de todos los niveles y disciplinas en el uso de los materiales, guías y textos sobre educación sexual” (GUATEMALA, 2018d, p. 76), principalmente en aras de disminuir el embarazo adolescente. Pero no hay mención sobre esta temática en la Política Pública y Plan de Acción para la Protección Integral a la Niñez y la Adolescencia (2017-2032) (GUATEMALA, 2018e).

En la Ley de Desarrollo Social de Guatemala, se habla en hacer accesibles los programas de educación sexual para fomentar estilos de vida saludables, para la prevención de embarazos no deseados y en temprana edad, así como prevención de infecciones de transmisión sexual, en todos los centros y niveles educativos del país (GUATEMALA, 2018f).

La Revisión Nacional Voluntaria hizo un análisis de los 17 Objetivos de Desarrollo Sostenible (ODS) y fue elaborado con la participación de instituciones públicas, consejos de desarrollo, cooperación internacional, sector empresarial, y sociedad civil. Los participantes indicaron que hace falta atender los problemas de educación sexual y planificación familiar para contribuir a la reducción de la pobreza en el país (GUATEMALA, 2019).

\section{Costa Rica}

En 2010 el gobierno de Costa Rica asumió la ausencia de una estrategia integral e intersectorial de educación de la sexualidad y que en aquel momento esta era responsabilidad de los padres y madres de familia y no del Estado, contribuyendo a una "educación de la sexualidad desactualizada, adultocéntrica, sexista, heterosexista y fundamentalista" (COSTA RICA, 2010, p. 27). Pero planteaba un modelo educacional amplio y diverso, en el marco de los derechos humanos (COSTA RICA, 2010).

La educación para la afectividad y sexualidad integral debe acompañar a las personas a lo largo del curso de vida, proveyendo oportunidades de aprendizaje, reflexión y revisión crítica que les permita identificar aquellos mitos, prejuicios, tabúes que limitan su comprensión y su vivencia, a la vez que amplía sus conocimientos y mejora sus actitudes para vivir la afectividad y la sexualidad con plenitud, placer, seguridad y responsabilidad en cada uno de estos momentos del desarrollo. (COSTA RICA, 2017, p. 09).

Esta definición hace parte del Programa de Estudios de Educación para la Afectividad y Sexualidad Integral del Ministerio de Educación Pública de Costa Rica, documento más reciente que sugiere metodologías participativas para la educación sexual. El programa está dividido en temas sobre derechos humanos, diversidades, interculturalidad, generacional-contextual, género e inclusión (COSTA RICA, 2017).

La enseñanza sobre sexualidad está garantizada, de manera no especificada o estratégica, en la Ley General de Salud (COSTA RICA, 2018a), en el Código de la Niñez y la Adolescencia (COSTA RICA, 2018b), en la Política Nacional para la Niñez y la Adolescencia (COSTA RICA, 2018c), en el Plan de Acción de la Política Nacional de Igualdad y Equidad de Género (COSTA RICA, 2018d), en la Ley General de Protección a la Madre Adolescente (COSTA RICA, 2018e), en las estrategias de capacitación para el abordaje del VIH/sida en niños, niñas y adolescentes en condición de vulne- 
rabilidad o que viven con el virus (COSTA RICA, 2018f), en la Agenda Nacional de la Niñez y la Adolescencia (COSTA RICA, 2015), así como en al marco curricular para la educación de la niñez desde el nacimiento hasta los seis años (COSTA RICA, 2018g). La Ley Fundamental de Educación no plantea el asunto (COSTA RICA, 2018h).

Es interesante destacar algunos documentos, como Creer y Crear la Costa Rica del Siglo XXI: Compromiso País, cuando habla en "priorizar una educación sexual comprensiva, laica y científica en todos los centros educativos del país" (COSTA RICA, 2018i, p. 21). Además, Costa Rica implementó métodos de acompañamiento a familias de estudiantes con discapacidad, donde asume la falta de atención a este público en materia de sexualidad y aborda informaciones acerca al cuerpo, sentimientos y manifestaciones sexuales de los alumnos y alumnas (COSTA RICA, 2016).

\section{Conclusión}

Los tres países investigados presentan en leyes y programas nacionales la necesidad de un abordaje y enseñanza de la sexualidad para niños, niñas y adolescentes. Entre tanto, México está atrás de lo que sugieren los mecanismos de derechos humanos, en relación al tema de que la educación en sexualidad debe ser planteada no solo para evitar embarazos o infecciones sexualmente transmisibles. Pero, también, para el pleno desarrollo del joven y para disminuir casos de violencia entre este grupo, incluyendo abusos, suicidios, violaciones y acoso escolar. México fue el primero en Latinoamérica por tratar el tema en la educación, pero actualmente enfrenta los problemas de haber abandonado esta práctica, al estar en la primera posición mundial en los casos de bullying. Un estudio podría ser hecho para investigar si las tasas de violencia en el país tienen relación con la intimidación que niños, niñas y adolescentes reciben y practican en temprana edad. Además, como abordado en este actual estudio, también se podrían analizar si estos casos de bullying afectan la salud mental y física de las víctimas en la fase adulta, causando daños en el sistema de salud, de seguridad social y, consecuentemente de acuerdo con el elevado número de casos, a la economía de la nación.

En la novena posición en el ranking, Guatemala sigue los mismos pasos de México, con un apéndice: reconoce la pluralidad de los beneficios de la educación sexual. Pero todavía no tiene un programa con la efectividad que el tema necesita, incluso con la forma transdisciplinaria sugerida por los órganos de derechos humanos, o sea, presente en diferentes fases de la educación, para alcanzar beneficios plurales.

Costa Rica está en la onceava posición en la clasificación, presentando uno de los peores índices de bullying del mundo. Pero, de acuerdo con los documentos analizados, reconoce la importancia de una educación en sexualidad para mejorar el tema, incluso con atención a grupos históricamente vulnerabilizados y una visión bastante próxima de los recomendados por entidades de derechos humanos internacionales que plantean el tema en convenciones recientes. Entre tanto, los resultados positivos pueden tardar en aparecer, pues todavía las acciones son recientes, en si tratando de un programa nacional. Los países latinoamericanos tienen una forma irregular de trabajar la educación sexual en los planes y leyes gubernamentales. Entre los tres analizados, Costa Rica hace intentos de un método de enseñanza transversal, y si eso se trabaja de manera efectiva, es muy probable que el país esté en mejor clasificación en el ranking futuro.

El actual estudio fue hecho basado en la tasa de bullying en los países mencionados, pero también se podrían clasificar otros temas relacionados con la educación sexual, como el suicidio, los asesinatos contra la población LGBTQI+, la violencia motivada por prejuicios, violencia y abuso sexual, violación de niños, niñas y adolescentes, embarazo adolescente, feminicidio, entre otros. En este contexto, la efectividad de las leyes en las escuelas desempeña un papel clave, al igual que las familias, en la identificación y ayuda a los jóvenes vulnerables o perjudicados por la falta de información, teniendo en cuenta que el acceso a la información es también un derecho humano. 
Seguir los parámetros en educación para la sexualidad presentados en las convenciones internacionales representa un paso para el desarrollo de una nación, sobre todo para las pobres, así como las que hacen parte de América Latina. Es importante resaltar que Brasil aparece en diversas clasificaciones de violencia, siendo la nación del mundo con más asesinatos de transexuales, quinto en feminicidios, onceavo en explotación sexual infantil y también aparece entre los primeros países latinos en casos de embarazo adolescente y el bullying. La aplicabilidad sugerida en las convenciones internacionales en un contexto nacional puede colaborar con cambios en ámbitos sociales y económicos. Investigar este argumento en la realidad brasileña, incluso con los intentos y dificultades de aplicabilidad de iniciativas de educación sexual, puede contribuir a una nueva mirada y propuesta educacional.

\section{Referencias}

ALMEIDA, Kaciane Daniella; LUZ, Nanci Stancki da. Educação Sexual, uma discussão para a escola? 1. ed. Curitiba: Appris, 2014.

ALVES, José Augusto Lindgren. Relações Internacionais e Temas Sociais. A Década das Conferências. 1. ed. Brasília: IBRI, 2001.

ASTUDILLO, María Nelcy Muñoz. La Educación Sexual en Latinoamérica: un campo de fuerzas en tensión. Revista Cultura del Ciudadano Enfermería, Colombia, v. 14, n. 1, p. 57-70, enero/feb. 2017. Disponible en: https://doi.org/10.18041/17945232/cultrua.2017v14n1.4329. Acceso en: 14 dic. 20.

BEDOYA ABELLA, Claudia Liliana. Educación para la sexualidad y construcción de ciudadanía. Sophia, Colombia, v. 10, n. 1, p. 95-106, enero/jun. 2014. Disponible en: https://www.redalyc.org/pdf/4137/413734078009.pdf. Acceso en: 18 dic. 20.

BRUÑOL, Miguel Cillero. El interés superior del niño en el marco de la Convención Internacional sobre los Derechos del Niño. In: OVIEDO, Mauricio González; ULATE, Elieth Vargas. Derechos de la niñez y la adolescencia. 1. ed. Costa Rica: Conamaj, 2001. p. 32-45. Disponible en: https://www.unicef.org/costarica/media/876/file/Derechos\%20de\%20 la\%20Ni\%C3\%B1ez\%20y\%20Adolescencia:\%20Antolog\%C3\%ADa.pdf. Acceso en: 17 nov. 20.

BSF. Estadísticas Mundiales de Bullying 2017/2018. Primer Trabajo Oficial en el Mundo contra el Bullying. In: ONG Internacional Bullying Sin Fronteras, [s. l.], junio 2020. Disponible en: https://bullyingsinfronteras.blogspot.com/2018/10/ estadisticas-mundiales-de-bullying_29.html. Acceso en: 14 nov. 20.

COSTA RICA. Política Nacional de Sexualidad 2010-2021. San José: Ministerio de salud, 2010. Disponible en: https:// www.siteal.iiep.unesco.org/sites/default/files/sit_accion_files/siteal_costa_rica_0711.pdf. Acceso en: 03 enero 2020.

COSTA RICA. Agenda Nacional de la Niñez y la Adolescencia. Metas y compromisos 2015-2021. San José: Consejo Nacional de Niñez y Adolescencia, 2015. Disponible en: https://www.siteal.iiep.unesco.org/sites/default/files/sit_accion_ files/10081.pdf. Acceso en: 03 enero 2020.

COSTA RICA. Acompañamiento a las familias de estudiantes con discapacidad en los centros educativos. Costa Rica: Ministerio de Educación Pública, 2016. Disponible en: https://www.siteal.iiep.unesco.org/sites/default/files/sit_accion_ files/10086.pdf. Acceso en: 03 enero 2020.

COSTA RICA. Programa de Estudios de Educación para la Afectividad y Sexualidad Integral. Costa Rica: Ministerio de Educación Pública, 2017. Disponible en: https:/www.mep.go.cr/sites/default/files/programadeestudio/programas/ afectividad-sexualidad-diversificada.pdf. Acceso en: 05 enero 2020.

COSTA RICA. Ley $n .^{\circ}$ 5.395/1973, de 30 de octubre de 1973. Ley General de Salud. Costa Rica: Ministerio de salud, 2018a. Disponible en: https://www.siteal.iiep.unesco.org/sites/default/files/sit_accion_files/siteal_costa_rica_0684. pdf. Acceso en: 05 enero 2020.

COSTA RICA. Ley n. ${ }^{\circ} 7.739$, de 6 de febrero de 1998. Código de la Niñez y la Adolescencia. Costa Rica: Poder legislativo, 2018b. Disponible en: https://www.siteal.iiep.unesco.org/sites/default/files/sit_accion_files/siteal_costa_rica_0673.pdf. Acceso en: 05 enero 2020. 
COSTA RICA. Política Nacional para la Niñez y la Adolescencia. Costa Rica: Patronato Institucional de la Infancia, 2018c. Disponible en: https://www.siteal.iiep.unesco.org/sites/default/files/sit_accion_files/siteal_costa_rica_0707. pdf. Acceso en: 05 enero 2020.

COSTA RICA. Plan de Acción de la Política Nacional de Igualdad y Equidad de Género. San José: Instituto Nacional de las Mujeres, 2018d. Disponible en: https://www.siteal.iiep.unesco.org/sites/default/files/sit_accion_files/siteal_costa_ rica_0722.pdf. Acceso en: 05 enero 2020.

COSTA RICA. Ley $n .^{\circ} 7735$ (modificada a partir de la ley 8312). Ley General de Protección a la Madre Adolescente. Costa Rica: Asamblea Legislativa de la República de Costa Rica, 2018e. Disponible en: https://www.siteal.iiep.unesco. org/sites/default/files/sit_accion_files/6337.pdf. Acceso en: 05 enero 2020.

COSTA RICA. Estrategias de capacitación para el abordaje del VIH/sida en niños, niñas y personas adolescentes en condición de vulnerabilidad o que viven con el virus. Costa Rica: Ministerio de Salud, 2018f. Disponible en:__ https:// www.siteal.iiep.unesco.org/sites/default/files/sit_accion_files/6341.pdf. Acceso en: 03 enero 2020.

COSTA RICA. Marco curricular para la educación de la niñez desde el nacimiento hasta los seis años. Costa Rica: Ministerio de Educación Pública, 2018g. Disponible en: https://www.siteal.iiep.unesco.org/sites/default/files/sit_accion_ files/10095.pdf. Acceso en: 01 enero 2020.

COSTA RICA. Ley n..$^{\circ}$ 2160, de 02 de octubre de 1957. Ley Fundamental de Educación. San José: Ministerio de Educación Pública, 2018h. Disponible en: https://www.siteal.iiep.unesco.org/bdnp/222/ley-2160-ley-fundamental-educacion. Acceso en: 01 enero 2020

COSTA RICA. Creer y Crear la Costa Rica del Siglo XXI: Compromiso País. Costa Rica: Poder Ejecutivo, 2018i. Disponible en: https://www.siteal.iiep.unesco.org/sites/default/files/sit_accion_files/siteal_costa_rica_0718.pdf. Acceso en: 01 enero 2020.

COLOMBIA. Bases del Plan Nacional de Desarrollo 2018-2022. Bogotá, D.C.: Departamento Nacional de Planeación, 2018. Disponible en: https://www.siteal.iiep.unesco.org/sites/default/files/sit_accion_files/co_8000.pdf. Acceso en: 12 nov. 2020.

DURÁN, Natalia Herrera. Las pruebas de Sergio. In: EL Espectador, Bogotá, 07 sept. 2014. Disponible en: https:// tinyurl.com/y3quowwz. Acceso en: 10 jun. 2019.

FRISCHEISEN, Luiza Cristina. Educação de gênero na escola previne feminicídios, dizem especialistas. [Entrevista cedida a] Andreia Verdélio. Agência Brasil, Brasília, DF, 06 set. 2018. Disponible en: https://agenciabrasil.ebc.com.br/ politica/noticia/2018-09/educacao-de-genero-na-escola-previne-feminicidios-dizem-especialistas. Acceso en: 20 dic. 2020.

GUARNIERI, Tathiana Haddad. Os Direitos das mulheres no Contexto Internacional - da criação da ONU (1945) à Conferência de Beijing (1995). Revista Eletrônica da Faculdade Metodista Granbery, Juiz de Fora, n. 8, p. 1-28, enero/ jun. 2010. Disponible en: http://re.granbery.edu.br/artigos/MzUx.pdf. Acceso en: 15 nov. 2020.

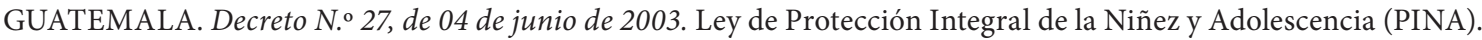
Guatemala: Poder Legislativo, 2018a. Disponible en: https://www.siteal.iiep.unesco.org/sites/default/files/sit_accion_files/ siteal_guatemala_0737.pdf. Acceso en: 07 dic. 2020.

GUATEMALA. Informe de la Estrategia de Articulación de los Objetivos de Desarrollo Sostenible al Plan y la Politica Nacional de Desarrollo K'atun: Nuestra Guatemala al 2032. Guatemala: Secretaría de Planificación y Programación de la Presidencia, 2018b. Disponible en: https://www.siteal.iiep.unesco.org/sites/default/files/sit_accion_files/10035. pdf. Acceso en: 07 dic. 2020.

GUATEMALA. K'atun: Nuestra Guatemala 2032. Guatemala: Consejo Nacional de Desarrollo Urbano y Rural, 2018c. Disponible en: https://www.siteal.iiep.unesco.org/sites/default/files/sit_accion_files/siteal_guatemala_0755.pdf. Acceso en: 07 dic. 2020.

GUATEMALA. Política Nacional de Promoción y Desarrollo Integral de las Mujeres y Plan de Equidad de Oportunidades 2008-2023. Guatemala: Secretaría Presidencial de la Mujer, 2018d. Disponible en: https://www.siteal.iiep.unesco.org/ sites/default/files/sit_accion_files/siteal_guatemala_0759.pdf. Acceso en: 07 dic. 2020.

GUATEMAlA. Política Pública y Plan de Acción para la Protección Integral a la Niñez y la Adolescencia (2017-2032). Guatemala: Comisión Nacional de la Niñez y de la Adolescencia, 2018e. Disponible en: https://www.siteal.iiep.unesco. org/sites/default/files/sit_accion_files/gt_6168.pdf. Acceso en: 07 dic. 2020.

GUATEMALA. Decreto N. 42/2001, de 26 de septiembre de 2001. Ley de Desarrollo Social. Guatemala: Poder Legislativo, 2018f. Disponible en: https://www.siteal.iiep.unesco.org/sites/default/files/sit_accion_files/gt_0742.pdf. Acceso en: 07 dic. 2020. 
GUATEMALA. Revisión Nacional Voluntaria 2019.2019. Guatemala: Secretaría de Planificación y Programación de la Presidencia, 2019. Disponible en: https://www.siteal.iiep.unesco.org/bdnp/3426/revision-nacional-voluntaria-2019. Acceso en: 07 dic. 20.

LONDOÑO, María Ladi E. Derechos Sexuales y Reproductivos. Los más humanos de todos los derechos. 1. ed. Cali: Talleres Gráficos de Impresora Feriva S.A. Cali, 1996.

MARTÍNEZ, Brenda; HERRERA, Fátima. Bullying: señales para identificarlo y qué hacer si su hijo es víctima de acoso. In: PRENSA Libre. Guatemala, 2 mayo 2020. Disponible en: https://www.prensalibre.com/vida/salud-y-familia/ bullying-senales-para-identificarlo-y-que-hacer-si-su-hijo-es-victima-de-acoso. Acceso en: 05 dic. 2020.

MEIER, Marcos; ROLIM, Jeanine. Bullying sem blá-blá-blá. 1. ed. Curitiba: Intersaberes, 2013.

MÉXICO. Modelo de Atención Integral Salud Sexual y Reproductiva para Adolescentes. México: Secretaría de Salud, $2018 \mathrm{a}$. Disponible en: https://www.siteal.iiep.unesco.org/sites/default/files/sit_accion_files/6528.pdf. Acceso en: 04 enero 2021.

MÉXICO. Estrategia Nacional de Atención a la Primera Infancia. México: SIPINNA, 2018b. Disponible en: https:// www.siteal.iiep.unesco.org/sites/default/files/sit_accion_files/6532_0.pdf. Acceso en: 04 enero 2021.

MÉXICO. Acuerdo N. 592/2011, de 19 de agosto de 2011. México: Diario Oficial de la Federación, 2018c. Disponible en: https://www.siteal.iiep.unesco.org/sites/default/files/sit_accion_files/mx_0078.pdf. Acceso en: 04 enero 2021.

MÉXICO. Estrategia Nacional para la Prevención del Embarazo en Adolescentes. México: Instituto Nacional de las Mujeres (INMUJERES), 2018d. Disponible en: https://www.siteal.iiep.unesco.org/sites/default/files/sit_accion_files/ mx_1151.pdf. Acceso en: 03 enero 2021.

MÉXICO. Ley General de Salud/1983, de 7 de febrero de 1984. México: Poder Legislativo, 2018e. Disponible en: https:// www.siteal.iiep.unesco.org/sites/default/files/sit_accion_files/mx_0092.pdf. Acceso en: 03 enero 2021.

MÉXICO. Estrategia Nacional de Educación Inclusiva. México: Secretaria de Educación Pública, 2018f. Disponible en: https://www.siteal.iiep.unesco.org/sites/default/files/sit_accion_files/10301.pdf. Acceso en: 03 enero 2021.

MÉXICO. Ley General de Educación, de 30 de septiembre de 2019. México: Diario Oficial de la Federación, 2019a. Disponible en: https://www.siteal.iiep.unesco.org/sites/default/files/sit_accion_files/10039_0.pdf. Acceso en: 04 enero 2021.

MÉXICO. Ley General de los Derechos de Niñas, Niños y Adolescentes, de 04 de diciembre de 2014 . Nueva Ley publicada en el Diario Oficial de la Federación, 2019b. Disponible en: https://www.siteal.iiep.unesco.org/sites/default/files/ sit_accion_files/10290.pdf. Acceso en: 04 enero 2021.

MÉXICO. Plan Nacional de Desarrollo 2019-2024. México: Gobierno de México, 2019c. Disponible en: https://www. siteal.iiep.unesco.org/sites/default/files/sit_accion_files/10042_0.pdf. Acceso en: 04 enero 2021.

MÉXICO. Agenda Digital Educativa. México: Secretaria de Educación Pública, 2019d. Disponible en: https://www. siteal.iiep.unesco.org/sites/default/files/sit_accion_files/10313.pdf. Acceso en: 04 enero 2021.

MÉXICO. Programa Sectorial de Educación 2020-2024. México: Secretaria de Educación Pública, 2020a. Disponible en: https://www.siteal.iiep.unesco.org/sites/default/files/sit_accion_files/3046.pdf. Acceso en: 04 enero 2021.

MÉXICO. Constitución Política de los Estados Unidos Mexicanos. México: Diario Oficial de la Federación el 05.02.1917. Última reforma publicada 18.12.2020.2020b. Disponible en: https://www.siteal.iiep.unesco.org/sites/default/files/ sit_accion_files/10288.pdf. Acceso en: 04 enero 2021.

MORALES-RAMIREZ, María Ester; VILLALOBOS-CORDERO, Margarita. The Impact of Bullying in the Integral Development and Learning from the Perspective of Children in Preschool and School. Educare, Costa Rica, v. 21, n. 3, p. 25-44, dic. 2017. Disponible en: http://dx.doi.org/10.15359/ree.21-3.2. Acceso en: 19 dic. 2020.

OLWEUS, Dan. Bullying at School. What We Know and What We Can Do. 1. ed. Oxford: Blackwell Publishing, 1993.

ORGANIZACIÓN DE LAS NACIONES UNIDAS (ONU). International Covenant on Economic, Social and Cultural Rights. United Nations, 1976. Disponible en: https://www.ohchr.org/EN/professionalinterest/pages/cescr.aspx. Acceso en: 13 dic. 2020.

ORGANIZACIÓN DE LAS NACIONES UNIDAS (ONU). Informe de la Conferencia Internacional sobre la Población y el Desarrollo. Organização das Nações Unidas, 1995. Disponible en: https://www.unfpa.org/sites/default/files/pub-pdf/ icpd_spa.pdf. Acceso en: 05 nov. 2020.

ORGANIZACIÓN DE LAS NACIONES UNIDAS (ONU). Informe de la Cuarta Conferencia Mundial sobre la Mujer. Organização das Nações Unidas, 1996. Disponible en: https://www.un.org/womenwatch/daw/beijing/pdf/Beijing\%20 full\%20report\%20S.pdf. Acceso en: 05 nov. 2020. 
ORGANIZACIÓN DE LAS NACIONES UNIDAS (ONU). Declaração Universal dos Direitos Humanos. Organização das Nações Unidas, 2009. Disponible en: https://nacoesunidas.org/wp-content/uploads/2018/10/DUDH.pdf. Acceso en: 15 nov. 2020.

ORGANIZACIÓN DE LAS NACIONES UNIDAS (ONU). Estrategia del PNUD para la Juventud 2014-2017. Organização das Nações Unidas, 2014. Disponible en: http://www.undp.org/content/dam/undp/library/Democratic\%20 Governance/Youth/UNDP-Youth-Strategy-2014-2017-SP.pdf. Acceso en: 23 nov. 2020.

ORGANIZACIÓN DE LAS NACIONES UNIDAS (ONU). International technical guidance on sexuality education. An evidence-informed approach. Organização das Nações Unidas, 2018. Disponible en: https://www.unaids.org/sites/ default/files/media_asset/ITGSE_en.pdf. Acceso en: 07 nov. 2020.

ORGANIZACIÓN DE LAS NACIONES UNIDAS (ONU). Informe del Consejo de Derechos Humanos. Organización de las Naciones Unidas, 2019. Disponible en: https://undocs.org/es/A/74/53. Acceso en: 13 nov. 2020.

ORGANIZACIÓN DE LOS ESTADOS AMERICANOS (OEA). Convención Interamericana para Prevenir, Sancionary Erradicar la Violencia contra la Mujer (Convención de Belém do Pará). Belém: Organización de los Estados Americanos, 1995. Disponible en: http://www.oas.org/es/mesecvi/docs/BelemDoPara-ESPANOL.pdf. Acceso en: 05 dic. 2020.

ORGANIZACIÓN MUNDIAL DE LA SALUD (OMS). Suicidio. Genebra: OMS, 2019. Disponible en: https://www. who.int/es/news-room/fact-sheets/detail/suicide. Acceso en: 05 nov. 2020.

PESCI EGUIA, Ana Lucía. Prevención del bullying en México: el caso de los niños y adolescentes sobredotados. Revista de El Colegio de San Luis, México, v. 5, n. 10, p. 104-133, jul./dic. 2015. Disponible en: http://www.scielo.org.mx/scielo. php?script=sci_arttext\&pid=S1665-899X2015000200104\&lng=es\&nrm=iso. Acceso en: 22 dic. 2020.

RAMÍREZ, Gabriela Rodríguez. Treinta años de educación sexual en México In: MíCHER, Martha Lucía (coord.). Población, desarrollo y salud sexual y reproductiva. México: Grupo Parlamentario del PRD Cámara de Diputados Congreso de la Unión LIX Legislatura, 2004. p. 13-28.

ROJAS, Rosalba; CASTRO, Filipa de; VILLALOBOS, Aremis et al. Educación sexual integral: cobertura, homogeneidad, integralidad y continuidad en escuelas de México. Salud Publica de México, México, v. 59, n. 1, p. 19-27, enero/ feb. 2017. Disponible en: https://doi.org/10.21149/8411. Acceso en: 04 enero 2021.

SAWYER, Donald. Palco e bastidores da Conferência Internacional sobre População e Desenvolvimento. Revista Brasileira de Estudos de População, Rio de Janeiro, v. 36, dez. 2019. Disponible en: https://doi.org/10.20947/s01023098a0091. Acceso en: 10 dez. 2020.

SPACCAROTELLA, Sabrina D. La Convención Interamericana para prevenir, sancionar y erradicar la violencia contra la mujer "Convención de Belém do Pará": La aplicación real y efectiva en el ámbito judicial argentino. Lex: Revista de la Facultad de Derecho y Ciencias Políticas, Peru, v. 16, n. 21, p. 53-78, abr. 2019. Disponible en: http://dx.doi.org/10.21503/ lex.v16i21.1541. Acceso en: 12 dic. 2020.

SCIOSCIOLI, Sebastián. El Derecho a la Educación Como Derecho Fundamental y Sus Alcances en el Derecho Internacional de Los Derechos Humanos. Journal of Supranational Policies of Education, España, n. 2, p. 6-24, jun. 2014. Disponible en: https://revistas.uam.es/jospoe/article/view/5625. Acceso en: 27 dic. 2020.

UNESCO. Orientações Técnicas Internacionais sobre Educação em Sexualidade. Organización de las Naciones Unidas para la Educación, la Ciencia y la Cultura, 2010. Disponible en: https://educacionsexual.mineduc.cl/docs/2010_\%20 UNESCO_OrientacionesEdSexualUnesco.pdf. Acceso en: 03 dic. 2020.

UNESCO. Educación integral en sexualidad para prevenir la violencia por razones de género. Organización de las Naciones Unidas para la Educación, la Ciencia y la Cultura, 2018a. Disponible en: https://es.unesco.org/news/educacion-integral-sexualidad-prevenir-violencia-razones-genero. Acceso en: 03 dic. 20.

UNESCO. Nuevos datos revelan que en el mundo uno de cada tres adolescentes sufre acoso escolar. Organización de las Naciones Unidas para la Educación, la Ciencia y la Cultura, 2018b. Disponible en: https://es.unesco.org/news/nuevos-datos-revelan-que-mundo-cada-tres-adolescentes-sufre-acoso-escolar. Acceso en: 03 dic. 2020.

UNICEF. Convenção sobre os Direitos da Criança. Fundo das Nações Unidas para a Infância, 1990. Disponible en: https://www.unicef.org/brazil/convencao-sobre-os-direitos-da-crianca. Acceso en: 13 dic. 2020.

Data de submissão: 06/01/2021

Data de aceite: $22 / 01 / 2021$ 